\title{
Personal Involvement of Residents and Development Strategies in a Community Planning
}

\author{
Takayuki Kumazawa*1 and Yoshiki Nakamura² $^{2}$
}

\author{
${ }^{1}$ Doctoral Student, Department of Built Environment, Tokyo Institute of Technology, Japan \\ ${ }^{2}$ Associate Professor, Department of Built Environment, Tokyo Institute of Technology, Japan
}

\begin{abstract}
This paper proposes a method that can be used in local government of the information society. By using this method, governments will be able to provide residents with information on infrastructure development projects efficiently. In this study the authors classified residents into types using Personal Involvement Inventory (PII) (Zaichkowsky, 1985) and examined their response to provided information. With the result of the experiment, the authors concluded that the tendencies of residents' consent to provided information differed according to type of resident classified by use of PII and to whether an evaluation was included in provided information. Base on our findings, electronic governments should measure the involvement of residents by use of PII when certifying the identity of residents who access the government over the Internet, and they should also provide residents with information through the method proposed.
\end{abstract}

Keywords: development strategies; information; personal involvement; community planning; consensus building

\section{Introduction}

In the information society, residents have the advantage to access, obtain, share, and utilize various information easily using the Internet, mobile phones, and broadband communication without restriction of time and place. Local governments are planning to make the utmost use of information technology and develop electronic government, in order to create a society where people can easily utilize information resources and accumulate knowledge. Some local governments have already begun to implement administration services such as information disclosure, administration evaluation, and electronic bidding through the Internet. It is expected that people will exchange information each other more actively as life becomes more convenient and comfortable.

However, in such information society, residents also face a disadvantage simultaneously; they have to deal with information in a complicated information environment. There is a possibility that people reach inappropriate information and acquire wrong knowledge due to lack of special technique or skill. Information

*Contact Author: Takayuki Kumazawa, Doctoral Student, Dep. of Built Environment, Tokyo Institute of Technology, 4259 Nagatsuta-cho, Midori-Ku, Yokohama, 226-8502 Japan Tel: +81-45-924-5618 Fax: +81-45-924-5574

e-mail: kumazawa@enveng.titech.ac.jp

(Received November 8, 2002 ; accepted March 10, 2003 ) can be misread, misused, or interpreted differently from person to person. The judgment of residents in a community is not absolute: residents' interpretations differ by time and circumstance, and differ even among residents. From the viewpoint of community planning, it is assumed that these factors can hinder the process of consensus building. Since currently employed methods of achieving consensus building are not always effective, it is important to implement a comprehensive method of strategic consensus building. It is also necessary to achieve an adequate understanding of present problems, the contents of plans, and how plans will affect society, the economy, the environment, and life in general in the community. In a sustainable society, it is important to find solutions that satisfy many residents and to establish satisfactory policies. Accordingly, to treat every resident's ideas and evaluations carefully, it is necessary to take residents' subjective evaluation into consideration. Local governments should therefore provide residents with information in an easy-tounderstand method that considers resident characteristics while also placing importance on information content.

This paper proposes a method that can be used in local government of the information society. By using this method, governments will be able to provide residents with information on infrastructure development projects efficiently. In this study the authors classified residents into types using PII (Zaichkowsky, 1985) and examined their response to provided information. 


\section{Theoretical Background}

The theoretical background of this study is the cognitive response approach in the field of research on communication and attitude. At first, psychologist Hovland and Janis, and Kelley (1953) began researching communication and attitude, and then Petty, Ostrom, and Brock (1981) tried systematizing the cognitive response approach. They thought that cognitive reactions, produced when a person is in the process of communication, would determine the direction and quantity of attitude change. In addition, they believed that various ideas, produced when a person receives a message, were also important. That is, when a person receives a message and acquires a new knowledge, $\mathrm{s} / \mathrm{he}$ interprets it in relation to his/her own knowledge system that $\mathrm{s} /$ he already has. In this process, ideas of approving or opposing the new knowledge arise in the person's mind. When plenty of ideas that are favorable to the message occur, the person is supposed to take a positive attitude. On the other hand, if many unfavorable ideas occur, the person is likely to take a defiant or repellent attitude.

Concerning the cognitive response approach, Petty and Cacioppo (1986) proposed two fundamental routes of information that connect with attitude change. One is the central route and the other is the peripheral route. The central route is influenced by the message itself. Messages can be divided into evaluation messages and non-evaluation messages. In this study, whether the information provided to subjects includes evaluation or not is used as an experimental parameter (Table 1). It is predicted that attitude change caused by information transmitted through the central route is sustainable, and the subject will be consistent in his/her attitude and behavior. Attitude change also depends on whether the message includes evaluation or not. On the other hand, the peripheral route is not influenced by the message itself, but by various additional information of the provided message (for example, the level of expertise of the source of information). It is expected that attitude change caused by information via the peripheral route will be temporary, and the subject will lose consistency in his/her behavior. Another parameter employed in this study is that whether the source of information involves a high expertise or not (Table 1).

Whether information passes the central or peripheral route is related to the strength of the motivation toward the object, and the motivation depends on how deep the person involves in the object. Involvement has been studied as a factor that determines caution distribution and processing level toward the object in researches on consumer behavior and advertising in the field of social psychology (Celsi and Olson, 1988; Chakin, 1980). In the study of advertising, involvement is used to manipulate a process in which a consumer reacts on the object, is personally influenced by the object, and is thereby motivated. However, involvement was measured by various means through the response toward a message, and the definition thereof also varied. Under these circumstances, Zaichkowsky (1985) proposed Personal Involvement Inventory (PII). In PII, personal involvement is defined as the person's relevance toward the object, based on his/her own needs, desires, values, and interests. In this study, the involvement of subjects toward community planning is measured by PII.

Table 1. Experimental Conditions

\begin{tabular}{|c|c|c|c|}
\hline & & $\begin{array}{l}\text { Evaluation-message } \\
\text { The evaluation message contains the same objective } \\
\text { description as the non-evaluation message together } \\
\text { with subjective opinions on how to improve the } \\
\text { present state and solve the problem concretely. }\end{array}$ & $\begin{array}{l}\text { Non-evaluation-message } \\
\text { The non-evaluation message contains an objective } \\
\text { description of the present state and a problem of the } \\
\text { town. }\end{array}$ \\
\hline 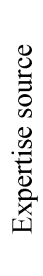 & $\begin{array}{l}\text { The experimenters describe } \\
\text { the following in the } \\
\text { beginning of the message. } \\
\text { [A professor who is an expert } \\
\text { in city planning described the } \\
\text { fundamental view on } \\
\text { community planning.] }\end{array}$ & $\begin{array}{l}\text { The problem with traffic is that many illegally- } \\
\text { parked cars and bicycles are disrupting the flow of } \\
\text { pedestrians, cars, and bicycles. I think they should } \\
\text { provide more parking lots to reduce illegal parking } \\
\text { on the street and secure pedestrians' safety. }\end{array}$ & $\begin{array}{l}\text { The problem with traffic is that many illegally- } \\
\text { parked cars and bicycles are disrupting the flow of } \\
\text { pedestrians, cars, and bicycles. }\end{array}$ \\
\hline 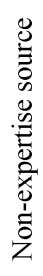 & $\begin{array}{l}\text { The experimenters describe } \\
\text { the following in the } \\
\text { beginning of the message. } \\
\text { [A high school student } \\
\text { described the fundamental } \\
\text { view on community planning } \\
\text { in a report.] }\end{array}$ & $\begin{array}{l}\text { The problem with traffic is that many illegally- } \\
\text { parked cars and bicycles are disrupting the flow of } \\
\text { pedestrians, cars, and bicycles. I think they should } \\
\text { provide more parking lots to reduce illegal parking } \\
\text { on the street and secure pedestrians' safety. }\end{array}$ & $\begin{array}{l}\text { The problem with traffic is that many illegally- } \\
\text { parked cars and bicycles are disrupting the flow of } \\
\text { pedestrians, cars, and bicycles. }\end{array}$ \\
\hline
\end{tabular}




\section{Experimental Procedure}

The procedure of the experiment is as follows. First, the authors explained to subjects about an information on a town, and then measured the subjects by PII- test. PII is a bipolar adjective scale and consists of 20 adjective pairs (Table 2). In this study, the object to be judged by the subjects is about community planning.

Second, the authors provided the subjects with an evaluation message from an expertise and non-expertise source, and a non-evaluation message from an expertise and non-expertise source. The non-evaluation message contains an objective description of the present state and a problem of the town. On the other hand, the evaluation message contains the same objective description as the non-evaluation message together with subjective opinions on how to improve the present state and solve the problem concretely. Furthermore, the authors compared the expertise source with the non-expertise source. In the expertise source conditions, the experimenters describe the following in the beginning of the message. [A professor who is an expert in city planning described the fundamental view on community planning.] In non-expertise source conditions, the experimenters describe the following in the beginning of the message. [A high school student described the fundamental view on community planning in a report.] Thus, the experimental condition consists of four patterns, namely, the expertise source or non-expertise source and the evaluation message or the non-evaluation message (Table 1).

Then, the subjects were evaluated by use of the affective response measures and the persuasiveness measures. The affective response measures consist of an affirmative measure and a negative measure. The persuasiveness measures consist of the validity of the opinion, the similarity of the opinion, and the liking to the source.

Finally, the authors tested the subjects by use of PII toward community planning again.

100 subjects who participated in the experiment had no relation to community planning. Each 25 subjects were assigned to one experimental condition at random, and the four experimental conditions were conducted.

\section{Results}

The authors examined the transition of the score of the PII-test between before and after showing a message to each subject. It is thought that high involvement leads to a high total score. The score varies from 140 to 20 . The personal involvement is rated as low when the score is lower than 70 points, as a little low when the score is in between 70 and 90 points, as a little high when the score is in between 90 and 110 points, and as high when the score is 110 or more points. Having investigated the changes of scores of all subjects, it was observed that there were three types of transition. The viewpoint from which the authors classified the transitions into three types is the transition of the score of the PII-test before
Table 2. Personal Involvement Inventory (PII)

\begin{tabular}{|ccc|}
\hline & community planning & \\
\hline important & - & unimportant \\
of no concern & - & of concern to me \\
irrelevant & - & relevant \\
means a lot to me & - & means nothing to me \\
useless & - & useful \\
valuable & - & worthless \\
trivial & - & fundamental \\
beneficial & - & not beneficial \\
matters to me & - & doesn't matter \\
uninterested & - & interested \\
significant & - & insignificant \\
vital & - & superfluous \\
boring & - & interesting \\
unexciting & - & exciting \\
appealing & - & unappealing \\
mundane & - & fascinating \\
essential & - & nonessential \\
undesirable & - & desirable \\
wanted & - & unwanted \\
not needed & - & needed \\
\hline
\end{tabular}

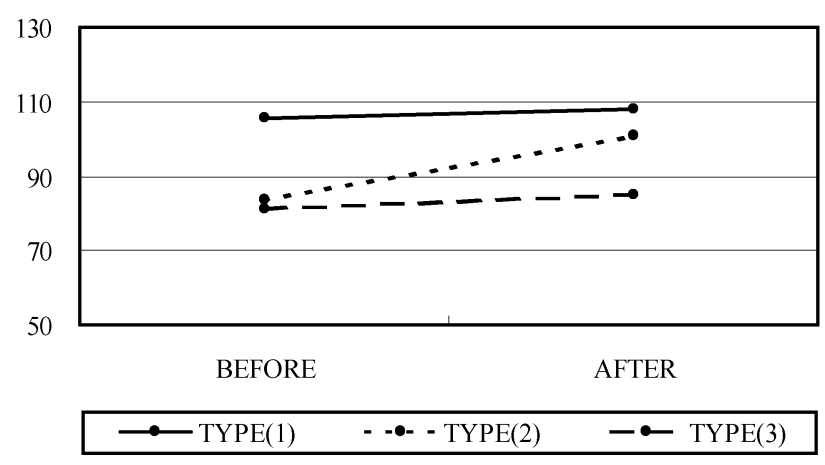

Fig.1. Average Value of PII-test in the Case of Expertise Source and Evaluation-message

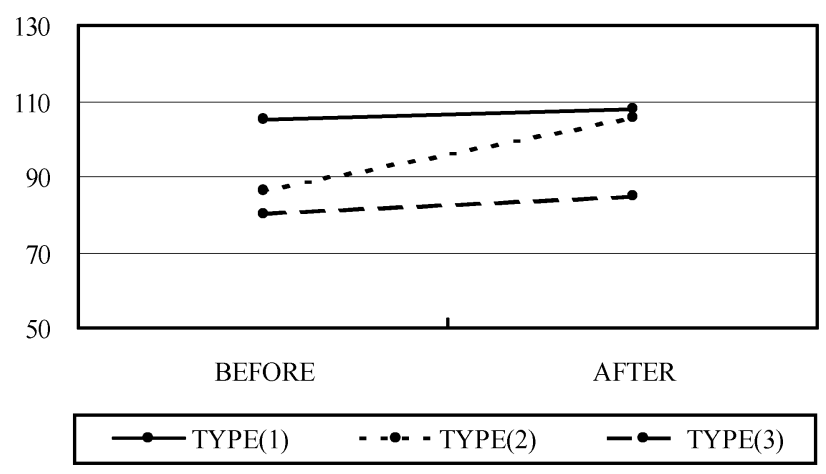

Fig.2. Average Value of PII-test in the Case of Expertise Source and Non-evaluation-message 
and after showing a message.

In type (1), the PII-test score is 90 points or more both before and after. Moreover, the difference between before and after scores is less than ten points. Thus, in type (1), personal involvement is high and makes a transition. In type (2), the PII-test score is lower before and 90 points or more after, and the difference between before and after scores is ten points or more. Thus, in type (2), personal involvement makes an upward transition. In type (3), the PII-test score is 89 points or less both before and after. Thus, in type (3), personal involvement is low and makes a transition.

Under these experimental conditions, the subjects are classified into three types. Figures 1 and 2 show the transition in the PII-test average score on the basis of an evaluation message from an expertise source and a nonevaluation message. The results in these two figures are clearly equivalent and the results under the four experimental conditions are nearly equal. This demonstrates the validity of classifying the subjects according to PII-test scores.

Next, after showing a message, the subjects evaluated the measures. Table 3 lists the average values of subject evaluation. The measures are affirmative affective response, negative affective response, opinion validity, opinion similarity, and liking of the source. It is considered that high evaluation leads to high score. The data are normally distributed so the analysis of variance (ANOVA) was utilized to assess any differences among the experimental conditions. The results were examined based on the data shown in Table 3.

First, when the data of $[\mathrm{A}]$ is compared with the data of [a], the data of [a] is higher at $\mathrm{P}<0.01$ level of significance, and when the data of $[\mathrm{B}]$ is compared with the data of [b], the data of [b] is lower at $\mathrm{P}<0.05$ level of significance. From these results, it appears that subjects of type (1) felt an affirmative affective response and negative affective response through the presentation of a non-evaluation message rather than of an evaluation message in the case of an expertise source. In addition, when the data of $[\mathrm{C}]$ is compared with the data of [c], the data of $[\mathrm{c}]$ is higher at $\mathrm{P}<0.05$ level of significance, and when the data of $[D]$ is compared with the data of [d], the data of [d] is higher at $\mathrm{P}<0.05$ level of significance. From these results, it appears that the subjects of type (1) responded to opinion validity and liking of the source through the presentation of a nonevaluation message rather than by presenting an evaluation message in the case of an expertise source. These results show that persons of high involvement could acquire a certain amount of knowledge through the presentation of a message and could imagine more. The authors considered, moreover, that they would accept a non-evaluation message and oppose an evaluation message. We have therefore demonstrated that the method of presenting a non-evaluation message affects persons of high involvement in community planning.

Second, when the data of [E] is compared with the

Table 3. The Average Values Subject Evaluation

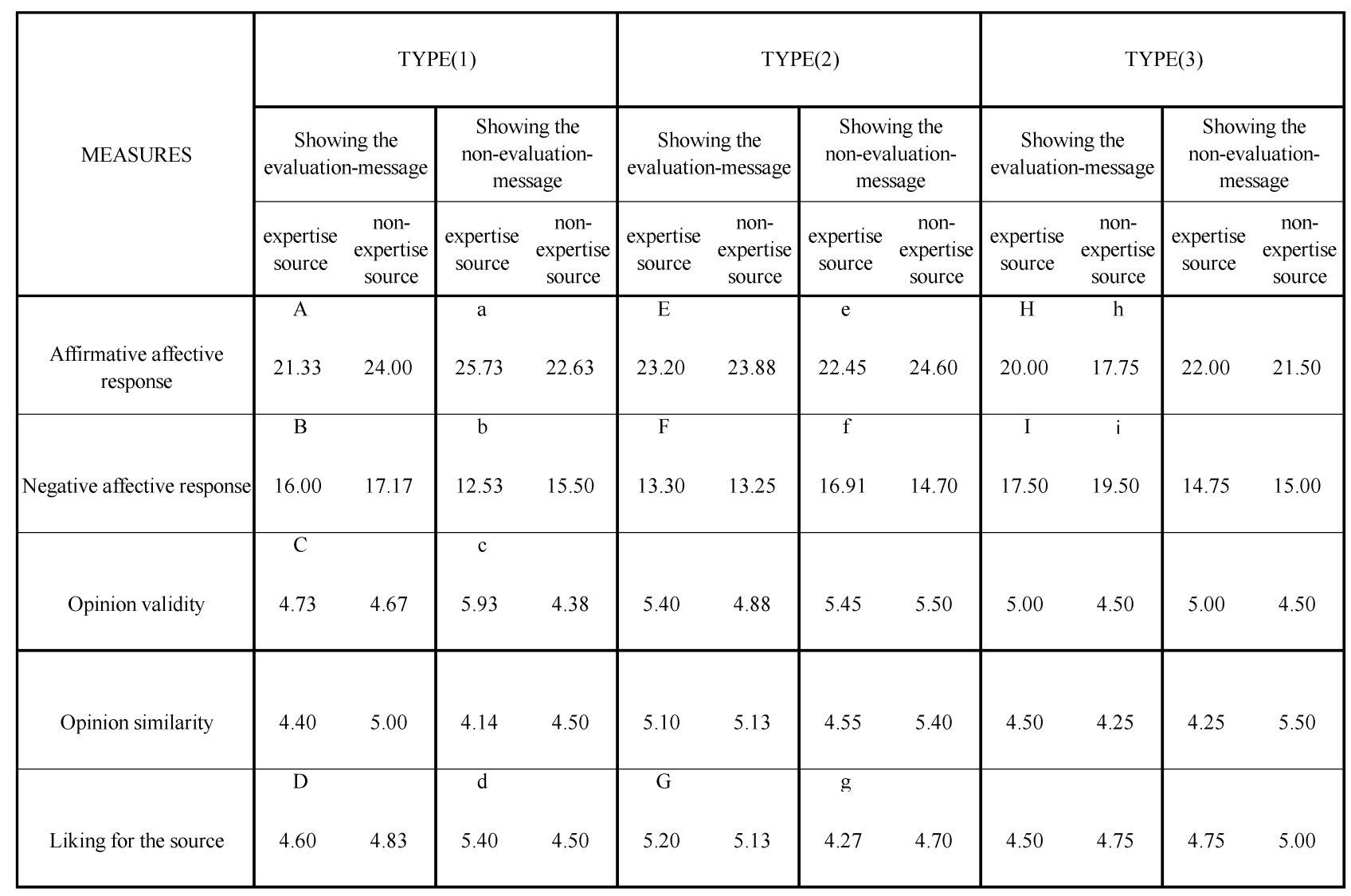


data of [e], the data of [E] is higher at $\mathrm{P}<0.05$ level of significance, and when the data of $[\mathrm{F}]$ is compared with the data of [f], the data of [f] is higher at $\mathrm{P}<0.05$ level of significance. From these results, it appears that subjects of type (2) felt an affirmative affective response and negative affective response through the presentation of an evaluation message rather than of a non-evaluation message in the case of an expertise source. In addition, when the data of [G] is compared with the data of $[\mathrm{g}]$, the data of $[\mathrm{G}]$ is higher at $\mathrm{P}<0.05$ level of significance. From these results, it appears that the subjects of type (2) responded to liking of the source through the presentation of an evaluation message rather than of a non-evaluation message in the case of an expertise source. These results show that persons of growing involvement could begin to acquire a certain amount of knowledge by presenting a message and make direct connections gradually. The authors considered, moreover, that they would accept an evaluation message easier than accepting a non-evaluation message. We have therefore demonstrated that the method of presenting an evaluation message affects persons of growing involvement in community planning.

Third, when the data of $[\mathrm{H}]$ is compared with the data of $[\mathrm{h}]$, the data of $[\mathrm{H}]$ is higher, and when the data of [I] is compared with the data of [i], the data of [I] is lower. From these results, it appears that subjects of type (3) felt an affirmative affective response and negative affective response in the case of an expertise source, although not with a significant difference. Since they did not have the materials to judge contents with, they would tend to be influenced by key information in the context of receiving a message instead of the content itself. It therefore appeared that an expertise source had an effect on persons of low involvement in community planning.

The authors confirmed that the tendencies of type (1) and type (2) coincided with the central route based on the cognitive reaction approach advocated by Petty and Cacioppo $(1981,1986)$, and that the tendency of type (3) coincided with the peripheral route.

\section{Conclusions}

In this study, the authors concluded that the tendencies of residents' consent to provided information differed according to type of resident classified by use of PII and to whether an evaluation was included in provided information. They demonstrated that the method of presenting a non-evaluation message affects persons of high involvement in community planning, and that the method of presenting an evaluation message affects persons of growing involvement in community planning. They also demonstrated that an expertise source affects persons of low involvement in community planning.

Therefore, in the early stages of infrastructure development projects, local governments should classify residents' type by use of PII and provide residents with information by the most suitable method according to whether residents' involvement is high or low. And, to increase the effect of this approach, local governments need to provide persons of high involvement with detailed, advanced, and objective information, and persons of low involvement with detailed, advanced, and subjective information.

From now on, local governments must offer services that are even more satisfying to residents. Furthermore, with the coming of the information age, local governments will probably become electronic governments. The authors should therefore apply the proposed method to electronic government systems. The aim of electronic government is to reconstruct local government based on an information infrastructure via the Internet. In this regard, there is currently much discussion about Internet security, and many technical developments are taking place in this area. For example, various types of individual authentication systems based on information infrastructures are being developed, as are electronic certification systems that authenticate electronic signatures (that play the role of traditional signatures or seals) and the person sending information. Of course, these developments are all very important, as they constitute a fundamental dimension concerning safety. Nevertheless, it also important in the information society to take the dimension of comfort into account in terms of the well-being of community residents.

Base on our findings, electronic governments should measure the involvement of residents by use of PII when certifying the identity of residents who access the government over the Internet. They should also provide residents with information through the method proposed. This is the strategy for development in the information society. It is expected to reduce friction and become a method of consensus building. This strategy should also raise the degree of resident satisfaction, find satisfactory resolutions to problems, and establish satisfactory policies.

\section{References}

1) Celsi, R.L. and Olson, J.C. (1988) The role of involvement in attention and comprehension processes, Journal of Consumer Research, 15, 210-224.

2) Chakin,S. (1980) Heuristics versus systematic information processing and the use of source versus message cues in persuasion, Journal of Personality and Social Psychology, 39, 752-766.

3) Hovland, C.I., Janis, I.L. and Kelley, H.H. (1953) Communication and persuasion, New Haven, CT.Yale University Press

4) Petty, R.E., Ostrom.T.M. and Brock, T.C.(Eds.), (1981) Cognitive responses in persuasion, Hillsdale,N.J., Lawrence Erlbaum Associates

5) Petty, R.E. and Cacioppo, J.T. (1986) Communication and persuasion, Central and peripheral routes to attitude change, New York, Springer Verlag

6) Zaichkowsky, J.L. (1985), Measuring the involvement construct, Journal of Consumer Research, 12, 341-352. 